which can be written as

$$
g^{\prime \prime}(x)+k^{2} g(x)=\frac{f^{i v}(x)-2 k^{2} f^{\prime \prime}(x)+k^{4} f(x)}{2 \lambda} .
$$

If we set $f(x) \equiv g(x)$ in (D) we obtain the Theorem IV. If $g(x)$ is a solution of the homogeneous equation

$$
g(x)=\lambda \int_{0}^{\infty} e^{-k|x-y|}|x-y| g(y) d y \quad x>0, k>0
$$

then $g(x)$ must satisfy the differential equation

$$
g^{i v}(x)-2\left(\lambda+k^{2}\right) g^{\prime \prime}(x)-k^{2}\left(2 \lambda-k^{2}\right) g(x)=0 .
$$

The real parts of the roots of the characteristic equation of the last differential equation have to be in absolute value less than $k$ in order that the integral may exist with $g(x)$ a solution of $(\mathrm{E})$. The complete discussion of the spectrum of characteristic values of this equation, as well as the treatment of certain integral equations of the second kind is reserved for another paper.

\title{
ON THE GENERAL EQUATIONS OF PROBLEMS OF AXIAL SYMMETRY IN THE THEORY OF PLASTICITY*
}

\section{BY P. S. SYMONDS (Brown University)}

1. Introduction. The solutions of axially symmetric problems in plasticity which have been presented in the literature depend in general upon the assumption of the so-called "full plasticity" condition. According to this assumption, two principal stress differences are taken equal to the yield stress in pure tension. This implies that two of the principal stresses are equal, and thus reduces the problem to a statically determinate one. On this basis solutions have been given for a number of important problems, such as those of the indentation of a rigid surface of revolution into a plastic mass (see for example papers of Hencky ${ }^{1}$ and Ishlinsky ${ }^{2}$ ).

This is a quite fictitious yield condition, with little physical or mathematical justification. Hence it is of interest to examine the general equations for axial symmetry using the well confirmed Mises flow condition, and, in particular, to determine whether or not these equations form a system of hyperbolic type. If so, the techniques of numerical integration using the networks of characteristic curves, which have been developed for supersonic fluid flow problems, could be applied to these problems of plasticity. Unfortunately the answer to this question, as shown in the present note, is that the equations are actually of elliptic type, the "characteristics" being real only under the special condition which reduces the problem to one of plane strain.

\footnotetext{
*Received July 8, 1948. The results presented here were obtained in the course of research conducted under a contract sponsored jointly by the Office of Naval Research and the Bureau of Ships.

${ }^{1} \mathrm{H}$. Hencky, Über einige statisch bestimmte Fälle des Gleichgewichts in plastischen Körpern, $\mathrm{Z}$. angew. Math. Mech. 3, 241-251 (1923).

${ }^{2} \mathrm{~A}$. Ishlinsky, The problem of plasticity with axial symmetry and Brinell's test, Prikl. Mat. Mekh. 8, 201-224 (1944).
} 
2. The general equations. In problems of axial symmetry defined by an axial coordinate $z$ and a radial coordinate $r$ we are concerned with four stress components $\sigma_{z}, \sigma_{r}, \sigma_{\theta}, \tau_{r z}$, and with two velocity components $u, w$. The two equations of equilibrium are

$$
\left.\begin{array}{l}
\frac{\partial \sigma_{r}}{\partial r}+\frac{\partial \tau_{r z}}{\partial z}+\frac{\sigma_{r}-\sigma_{\theta}}{r}=0 \\
\frac{\partial \sigma_{z}}{\partial z}+\frac{\partial \tau_{r z}}{\partial r}+\frac{\tau_{r z}}{r}=0 .
\end{array}\right\}
$$

The Mises yield condition becomes

$$
\left(\sigma_{r}-\sigma_{\theta}\right)^{2}+\left(\sigma_{\theta}-\sigma_{z}\right)^{2}+\left(\sigma_{z}-\sigma_{r}\right)^{2}+6 \tau_{r z}^{2}=6 K^{2},
$$

where $K$ is the yield stress in pure shear. The problem is evidently not statically determinate, so we must add the stress-strain relations to the foregoing. According to the Saint Venant-Levy-Mises flow theory ${ }^{3}$

$$
\left.\begin{array}{c}
e_{r}=\frac{\partial u}{\partial r}=\frac{1}{3} \lambda\left(2 \sigma_{r}-\sigma_{\theta}-\sigma_{z}\right), \\
e_{\theta}=\frac{u}{r}=\frac{1}{3} \lambda\left(2 \sigma_{\theta}-\sigma_{z}-\sigma_{r}\right), \\
e_{z}=\frac{\partial w}{\partial z}=\frac{1}{3} \lambda\left(2 \sigma_{z}-\sigma_{r}-\sigma_{\theta}\right), \\
g_{r z}=\frac{1}{2}\left(\frac{\partial u}{\partial z}+\frac{\partial w}{\partial r}\right)=\lambda \tau_{r z},
\end{array}\right\}
$$

where $e_{r}, e_{\theta}, e_{s}, g_{r z}$ are (tensor) strain-rate components (taken with respect to an arbitrary base, not necessarily time), and $\lambda(r, z)$ is a scalar point function depending on the material and on the state of stress at a generic point. We then have seven equations for the seven unknowns $\left(\sigma_{r}, \sigma_{\theta}, \sigma_{z}, \tau_{r_{z}}, \lambda, u, w\right)$.

Further work is facilitated by changing notation as follows

$$
\begin{aligned}
\sigma_{r} & =\omega+\xi, \\
\sigma_{\theta} & =\omega+\eta, \\
\sigma_{z} & =\omega+\zeta=\omega-(\xi+\eta), \\
\tau_{r z} & =\tau,
\end{aligned}
$$

where $\omega \equiv\left(\sigma_{r}+\sigma_{\theta}+\sigma_{z}\right) / 3$, and $\xi, \eta, \zeta$ are the "stress deviation". components. In the following, all subscripts indicate partial differentiation. The equations become:

3See for the instance William Prager, The stress-strain laws of the mathematical theory of plasticitya survey of recent progress, J. Appl. Mech. 15, 226-234 (1948). 
$\underset{\text { Equilibrium }}{\text { condition }}\left\{\begin{array}{l}\omega_{r}+\xi_{r}+\tau_{z}=\frac{1}{r}(\eta-\xi), \\ \omega_{z}-\xi_{z}-\eta_{z}+\tau_{r}=-\frac{1}{r} \tau,\end{array}\right.$

Yield

conditions

$$
\left\{\xi^{2}+\eta^{2}+\xi \eta+\tau^{2}=K^{2}\right.
$$

Stress-strain
relations $\left\{\begin{aligned} u_{r} & =\lambda \xi, \\ u & =r \lambda \eta, \\ w_{z} & =-\lambda\left(\xi^{\prime}+\eta\right), \\ u_{z}+w_{r} & =2 \lambda \tau .\end{aligned}\right.$

The four stress deviation components and $\lambda$ are determined by the five equations consisting of Eqs. (5) and two equations of compatibility derived from Eqs. (6). The compatibility equations may be written in the form

$$
\left.\begin{array}{rl}
(\lambda \xi)_{z z} & =2(\lambda \tau)_{r z}+(\lambda \xi+\lambda \eta)_{r r} \\
r(\lambda \eta)_{z z} & =2(\lambda \tau)_{z}+(\lambda \xi+\lambda \eta)_{r}
\end{array}\right\}
$$

as may be verified by substitution in (6).

3. Investigation of characteristic curves. We now investigate the possibility of the existence of characteristic curves $C$, on which

$$
z=z(r)
$$

such that on $C$ the second derivatives of the unknowns $(\omega, \xi, \eta, \tau, \lambda)$ are undefined although the functions themselves and all their first derivatives are defined. We first eliminate $\omega=\left(\sigma_{r}+\sigma_{\theta}+\sigma_{z}\right) / 3$ between Eqs. (5a) and (5b), obtaining

$$
2 \xi_{r z}+\eta_{r z}-\tau_{r r}+\tau_{z z}=\frac{1}{r}\left(\eta_{z}-\xi_{z}+\tau_{r}-\frac{1}{r} \tau\right) .
$$

We next differentiate Eq. (5c) twice with respect to $z$, obtaining

$$
(2 \xi+\eta) \xi_{z z}+(\xi+2 \eta) \eta_{z z}+2 \tau \tau_{z z}=-2\left(\xi_{z}^{2}+\xi_{z} \eta_{z}+\eta_{z}^{2}+\tau_{z}^{2}\right) .
$$

The justification for this is that we are investigating possible discontinuities in second derivatives, hence we are interested only in how Eq. (5c) defines the relations between second derivatives. Finally, we write out Eqs. (7) and obtain equations on the left sides of which only second derivatives and the functions themselves appear:

$$
\begin{gathered}
\lambda \xi_{r r}-\lambda \xi_{z z}+\lambda \eta_{r r}+2 \lambda \tau_{r z}+(\xi+\eta) \lambda_{r r}+2 \tau \lambda_{r z}-\xi \lambda_{z z} \\
=-2\left(\lambda_{r} \xi_{r}-\lambda_{z} \xi_{z}+\lambda_{r} \eta_{r}+\lambda_{r} \tau_{z}+\lambda_{z} \tau_{r}\right), \\
\lambda \eta_{z z}+\eta \lambda_{z z}=-2 \lambda_{z} \eta_{z}+\frac{1}{r}\left[2(\lambda \tau)_{z}+(\lambda \xi+\lambda \eta)_{r}\right] .
\end{gathered}
$$


Noting that a function $\varphi(r, z)$ given on a curve $C$ [defined as $z=z(r)$ ] is a function of $r$ only, on $C$ we have

$$
\frac{d \varphi}{d r}=\varphi_{r}+P \varphi_{z} ; \quad \varphi_{r}=\frac{d \varphi}{d r}-P \varphi_{z}
$$

where $P=d z / d r$. In particular if $\varphi=\xi_{z}$ or $\xi_{r}$ we obtain, respectively:

$$
\begin{aligned}
& \xi_{z r}=\frac{d \xi_{z}}{d r}-P \xi_{z z}, \\
& \xi_{r r}=\frac{d \xi_{r}}{d r}-P \frac{d \xi_{z}}{d r}+P^{2} \xi_{z z} .
\end{aligned}
$$

In like manner the second derivatives of all the functions appearing in Eqs. (9) may. be written in terms of the double derivatives with respect to $z$, and the parameter $P=d z / d r$ of the curve $C$. Equations (9) then take the form

$$
\left.\begin{array}{l}
A \xi_{z z}+B \eta_{z z}+C \tau_{z z}+D \lambda_{z z}=K, \\
A^{\prime} \xi_{z z}+B^{\prime} \eta_{z z}+C^{\prime} \tau_{z z}+D^{\prime} \lambda_{z z}=K^{\prime}, \\
A^{\prime \prime} \xi_{z z}+B^{\prime \prime} \eta_{z z}+C^{\prime \prime} \tau_{z z}+D^{\prime \prime} \lambda_{z z}=K^{\prime \prime}, \\
A^{\prime \prime \prime} \xi_{z z}+B^{\prime \prime \prime} \eta_{z z}+C^{\prime \prime \prime} \tau_{z z}+D^{\prime \prime \prime} \lambda_{z z}=K^{\prime \prime \prime}
\end{array}\right\}
$$

where $A, B, \cdots, D^{\prime \prime \prime}$ contain only the functions and the parameter $P$. The "characteristic condition" of the system is thus the equation:

$$
\left|\begin{array}{llll}
A & B & C & D \\
A^{\prime} & B^{\prime} & C^{\prime} & D^{\prime} \\
A^{\prime \prime} & B^{\prime \prime} & C^{\prime \prime} & D^{\prime \prime} \\
A^{\prime \prime \prime} & B^{\prime \prime \prime} & C^{\prime \prime \prime} & D^{\prime \prime \prime}
\end{array}\right|=0
$$

Determining the coefficients $A, B, \cdots, D^{\prime \prime \prime}$ for the system (9), we obtain the result:

$$
\left|\begin{array}{cccc}
-2 P & -P & -P^{2}+1 & 0 \\
2 \xi+\eta & \xi+2 \eta & 2 \tau & 0 \\
\lambda\left(P^{2}-1\right) & \lambda P^{2} & -2 \lambda P & (\xi+\eta) P^{2}-2 \tau P-\xi \\
0 & \lambda & 0 & \eta
\end{array}\right|=0 .
$$

This is a fourth degree algebraic equation in $P$, which reduces to

$$
\left[\left(P^{2}-1\right)(2 \xi+\eta)-4 \tau P\right]^{2}=-3\left[\eta\left(P^{2}+1\right)\right]^{2} .
$$


The four roots are

$$
P=\frac{d z}{d r}=\frac{\tau \pm K}{\xi+\eta\left(1 \pm i 3^{1 / 2}\right) / 2}
$$

where the \pm signs are independent; the yield equation in the original form of Eq. (5c) has been used in obtaining (12).

4. Conclusion. It is clear that real characteristics do not exist in general, since $P$ can be real only when $\eta=\sigma_{\theta}-\omega=0$. But when $\eta=0$ and $u \neq 0$ the stress equations become identical with the three equations for plane strain, under the Mises yield condition; the characteristic parameter $P$ then reduces to

$$
P=\frac{\tau \pm K}{\xi}
$$

As may be easily verified, this is the standard result for the slope of the characteristic curves in problems of plane plastic flow ${ }^{4}$, which provides a useful check on the present results.

An analogous but simpler investigation of Eqs. (6) leads to the conclusion that there are also no curves on which the velocity derivatives are undefined, even though the values of $u, w$ and the stresses are defined.

${ }^{4}$ See, for example, William Prager, Plasticity for the aerodynamicist, J. Aero. Sci. 15, 253-262 (1948).

\section{EFFECT OF HYPERBOLIC NOTCHES ON THE STRESS DISTRIBUTION IN A WOOD PLATE*}

\section{By C. BASSEL SMITH, University of Florida}

1. Introduction. If from an orthotropic material a flat plate is cut parallel to a plane of elastic symmetry, it will have two perpendicular axes of symmetry in the plane of the plate. Such a plate is said to be orthotropic. ${ }^{1}$ An example of this kind of plate is a plain-sawn board. In the discussion that follows, an orthotropic plate is assumed to be bounded on two sides by hyperbolic notches given by the equation $y^{2} / a^{2}-x^{2} / b^{2}=1$, and indefinitely extended in the other direction. The $x$ - and $y$-axes are taken parallel to the axes of symmetry. The plate is subjected only to forces directed parallel to the $x$-axis and applied in the plane of the plate. These forces are assumed to act at great distances from the $y$-axis, and in such a way that the traction over any cross-section perpendicular to the $x$-axis is statically equivalent to a single force of magnitude $P$ directed along the $x$-axis. The problem will be treated as one of plane stress.

2. The stress distribution in a wood plate with hyperbolic notches. The components of stress and strain in the orthotropic plate described are connected by the following relations ${ }^{2}$ :

${ }^{*}$ Received June 11, 1948.

${ }^{1}$ A. T. Price, Phil. Trans. (A) 228, 1-62 (1928). H. W. March, Physics, 7, 32-41 (1936). U. S. Forest Products Laboratory Reports Nos. 1300, 1301, 1304, 1312, 1316.

1. ' ${ }^{2}$ U. S. Forest Products Laboratory Report No. 1503. Love's notations for the stress and strain components are used, (A. E. H. Love, The mathematical theory of elasticity, 4th ed., Cambridge University Press, Cambridge, 1927). 